\title{
Adverse prognostic impact of the CpG island methylator phenotype in metastatic colorectal cancer
}

\author{
Yongjun Cha ${ }^{1,6}$, Kyung-Ju Kim ${ }^{2,6}$, Sae-Won Han ${ }^{* 1,3}$, Ye Young Rhee ${ }^{2}$, Jeong Mo Bae ${ }^{2}$, Xianyu Wen ${ }^{2}$, \\ Nam-Yun Cho ${ }^{2}$, Dae-Won Lee ${ }^{1}$, Kyung-Hun Lee ${ }^{1}$, Tae-Yong Kim ${ }^{1}$, Do-Youn Oh ${ }^{1,3}$, Seock-Ah Im ${ }^{1,3}$, \\ Yung-Jue Bang ${ }^{1,3}$, Seung-Yong Jeong ${ }^{4}$, Kyu Joo Park ${ }^{4}$, Gyeong Hoon Kang ${ }^{\star 2}$ and Tae-You Kim ${ }^{1,3,5}$ \\ ${ }^{1}$ Department of Internal Medicine, Seoul National University Hospital, Seoul, South Korea; ${ }^{2}$ Department of Pathology, Seoul \\ National University College of Medicine, Seoul, South Korea; ${ }^{3}$ Cancer Research Institute, Seoul National University College of \\ Medicine, Seoul, South Korea; ${ }^{4}$ Department of Surgery, Seoul National University Hospital, Seoul, South Korea and ${ }^{5}$ Department \\ of Molecular Medicine and Biopharmaceutical Sciences, Graduate School of Convergence Science and Technology, Seoul \\ National University, Seoul, South Korea
}

Background: The association between the $\mathrm{CpG}$ island methylator phenotype (CIMP) and clinical outcomes in metastatic colorectal cancer remains unclear. We investigated the prognostic impact of CIMP in patients with metastatic colorectal cancer treated with systemic chemotherapy.

Methods: Eight CIMP-specific promoters (CACNA1G, IGF2, NEUROG1, RUNX3, SOCS1, CDKN2A, CRABP1, and MLH1) were examined. The CIMP status was determined by the number of methylated promoters as high $(\geqslant 5)$, low $(1-4)$, and negative $(0)$.

Results: A total of 153 patients were included (men/women, 103/50; median age, 61 years; range, 22-80 years). The CIMP status was negative/low/high in 77/ 69/7 patients, respectively. Overall survival (OS) was significantly different among the three CIMP groups, with median values of 35.7, 22.2, and 9.77 months for the negative, low, and high groups, respectively $(P<0.001)$. For patients treated with fluoropyrimidine and oxaliplatin first-line chemotherapy $(N=128), O S$ and progression-free survival (PFS) were significantly different among the three CIMP groups; the median OS was 37.9, 23.8, and 6.77 months for the negative, low, and high groups, respectively $(P<0.001)$, while the median PFS was 9.97, 7.87 , and 1.83 months, respectively $(P=0.002)$. Response rates were marginally different among the three CIMP groups ( $53.4 \%$ vs $45.1 \%$ vs $16.7 \%$, respectively; $P=0.107$ ). For patients treated with fluoropyrimidine and irinotecan second-line chemotherapy $(N=86)$, only OS showed a difference according to the CIMP status, with median values of 20.4, 13.4, and 2.90 months for the negative, low, and high groups, respectively $(P<0.001)$.

Conclusions: The CIMP status is a negative prognostic factor for patients with metastatic colorectal cancer treated with chemotherapy.

Colorectal cancer (CRC) represents one of the major types of cancer and accounts for a high annual mortality rate worldwide (Siegel et al, 2014). Approximately 20\% patients with CRC present with distant metastasis at the time of diagnosis, and approximately $10 \%$ patients with early-stage disease eventually develop metastatic disease, which leads to death in many (Siegel et al, 2014).
The CpG island methylator phenotype (CIMP) is one of the key molecular alterations of CRC that exhibits extensive methylation in promoter CpG island regions (Hughes et al, 2013). CIMP-high CRC accounts for 7-29\% and 6-14\% cases in Western and Eastern populations, respectively (Kim and Kang, 2014), and has been associated with distinct clinicopathological characteristics,

\footnotetext{
*Correspondence: Professor S-W Han; E-mail: saewon1@snu.ac.kr or Professor GH Kang; E-mail: ghkang@snu.ac.kr

${ }^{6}$ These authors contributed equally to this work and share co-first authorship.
}

Received 11 March 2016; revised 6 May 2016; accepted 16 May 2016; published online 16 June 2016

(c) 2016 Cancer Research UK. All rights reserved 0007-0920/16 
including older age, female sex, proximal tumour location, poorly differentiated histology, BRAF mutations, and microsatellite instability (MSI) (Ogino et al, 2009).

With regard to the prognostic significance of CIMP in CRC, overall negative associations between the CIMP status and clinical outcomes have been reported in studies including patients with early and locally advanced disease (Van Rijnsoever et al, 2003; Shen et al, 2007; Ogino et al, 2007b; Iacopetta et al, 2008; Kim et al, 2009, 2011; Bae et al, 2011, 2013; Jover et al, 2011; Min et al, 2011; Sadanandam et al, 2013, 2014; Kim and Kang, 2014; MartinezGarcia et al, 2014; Shiovitz et al, 2014; Sistigu et al, 2014). A recent meta-analysis of 19 studies also showed that the CIMP status is independently associated with significantly decreased disease-free survival and overall survival (OS) in patients with CRC (Juo et al, 2014). Therefore, current knowledge suggests that the CIMP status is associated with adverse outcomes, particularly in patients with early and locally advanced CRC. However, the studies included in the meta-analysis were very heterogeneous with regard to patient characteristics, including chemotherapy regimens and MSI status, and methodologies, such as CIMP status definitions and methylation analyses.

However, the prognostic significance of CIMP in metastatic CRC has not been clearly defined. Most CIMP studies included patients with stage 1-4 disease, with a limited number of patients with stage 4 disease. Moreover, among the studies included in the meta-analysis, only one specifically analysed the CIMP status in patients with stage 4 CRC (Ogino et al, 2007b). Metastatic CRC differs from early and locally advanced disease in that chemotherapy has a central role in management. Furthermore, other patient characteristics, including the extent of metastasis and the performance status, affect the prognosis of these patients. Therefore, the prognostic role of CIMP in early and locally advanced disease cannot be directly extrapolated to metastatic CRC. To elucidate this role, studies focusing on patients with metastatic disease are necessary.

Therefore, we conducted the present study to evaluate the clinical implications of CIMP in patients with metastatic CRC treated with palliative chemotherapy by determining the relationship between the CIMP status and clinicopathological characteristics and clinical outcomes, including survival and response to chemotherapy.

\section{PATIENTS AND METHODS}

Patients and systemic chemotherapy regimens. The eligibility criteria for this retrospective study were as follows: diagnosis of metastatic colorectal adenocarcinoma, sporadic cancer without a family history, age $>18$ years, initiation of palliative systemic chemotherapy at Seoul National University Hospital (SNUH) from January 2009 to December 2012, adequate organ function, and donation of surgical tissue to the SNUH Tumor Bank. The study protocol was reviewed and approved by the Institutional Review Board of SNUH. The study was conducted in accordance with the recommendations of the Declaration of Helsinki for biomedical research involving human subjects.

Patients were treated with one of the following systemic chemotherapy regimens: FOLFOX [oxaliplatin $\left(85 \mathrm{mg} \mathrm{m}^{-2}\right.$ intravenous; i.v.), leucovorin ( $400 \mathrm{mg} \mathrm{m}^{-2}$ i.v.), and 5-fluorouracil $\left(400 \mathrm{mg} \mathrm{m}^{-2}\right.$ i.v. bolus followed by $2400 \mathrm{mg} \mathrm{m}^{-2}$ continuous i.v. over $46 \mathrm{~h}$ ) every 2 weeks], XELOX [oxaliplatin $\left(130 \mathrm{mg} \mathrm{m}^{-2}\right.$ i.v. on day 1) and capecitabine $\left(1000 \mathrm{mg} \mathrm{m}^{-2}\right.$ p.o. twice a day for 2 weeks) every 3 weeks], SOX [oxaliplatin $\left(130 \mathrm{mg} \mathrm{m}^{-2}\right.$ i.v. on day 1) and S-1 ( $40 \mathrm{mg} \mathrm{m}^{-2}$ twice a day for 2 weeks) every 3 weeks], FOLFIRI [irinotecan $\left(180 \mathrm{mg} \mathrm{m}^{-2}\right.$ i.v.), leucovorin $\left(200 \mathrm{mg} \mathrm{m}^{-2}\right.$ i.v. on days 1 and 2 ), and 5 -fluorouracil $\left(400 \mathrm{mg} \mathrm{m}^{-2}\right.$ i.v. bolus followed by $600 \mathrm{mg} \mathrm{m}^{-2}$ continuous i.v. over $22 \mathrm{~h}$ on days 1 and 2) every 2 weeks], XELIRI [irinotecan $\left(250 \mathrm{mg} \mathrm{m}^{-2}\right.$ i.v. on day 1$)$ and capecitabine ( $1000 \mathrm{mg} \mathrm{m}^{-2}$ p.o. twice a day for 2 weeks) every 3 weeks], and capecitabine alone [capecitabine $\left(1250 \mathrm{mg} \mathrm{m}^{-2}\right.$ p.o. twice a day for 2 weeks) every 3 weeks]. At the physician's discretion, bevacizumab $\left(5 \mathrm{mg} \mathrm{kg}^{-1}\right.$ i.v. on day 1 of each cycle of FOLFOX/FOLFIRI or $7.5 \mathrm{mg} \mathrm{kg}^{-1}$ i.v. on day 1 of each cycle of XELOX/XELIRI) or cetuximab $\left(400 \mathrm{mg} \mathrm{m}^{-2}\right.$ i.v. on day 1 and $250 \mathrm{mg} \mathrm{m}^{-2}$ on day 8 and weekly thereafter) was combined with cytotoxic chemotherapy. Computed tomography (CT) was performed every four cycles for biweekly regimens and three cycles for three-weekly regimens during the chemotherapy period or earlier if disease progression was suspected. Disease progression was defined on the basis of the CT findings.

Methylation analyses. Only primary tumours were subjected to methylation, mutation, and microsatellite analyses. Analysis of DNA methylation was performed as previously described (Kang et al, 2008; Kim et al, 2009). Tumour DNA samples were bisulphite-modified using the EZ DNA methylation kit (Zymo Research, Orange, CA, USA) and analysed for methylation in eight CIMP-specific CpG island loci (CACNA1G, IGF2, NEUROG1, RUNX3, SOCS1, CDKN2A, CRABP1, and MLH1) using a methylation-specific, probe-based, real-time PCR method (the MethyLight assay). The PCR conditions have been described previously (Weisenberger et al, 2006; Kang et al, 2008; Kim et al, 2009). The detailed primer information is provided in Supplementary Material 1 . The methylation status at each locus was reported as a percentage of methylated reference (PMR) (Kim et al, 2009). The MethyLight assay was repeated in triplicate, and of the three measured values, the median was considered the representative value for the methylation level of each marker. Each marker was considered methylated when the median PMR was $>4$. The CIMP status was considered high when $\geqslant 5$ methylated promoters were identified, low when 1-4 methylated promoters were identified, and negative when no methylated promoters were identified (Ogino et al, 2006b; Kim et al, 2009; Han et al, 2013).

Mutation and microsatellite analyses. BRAF mutations at codon 600 (V600E) were analysed using pyrosequencing. The microsatellite status was studied by evaluating five microsatellite markers (D2S123, D5S346, D17S250, BAT25, and BAT26), as previously described (Han et al, 2013; Lee et al, 2015). Either forward or reverse primers for each marker were labelled with fluorescence, and the PCR products were subjected to electrophoresis and analysed. The MSI status was classified as follows: high (instability at $\geqslant$ two microsatellite markers), low (instability at one marker), and microsatellite stable (MSS). Only patients with the MSI-high status were considered positive for MSI, while those with the MSIlow status and MSS were grouped together.

Statistical analysis. The primary aim of the present study was to determine the effects of the CIMP status on clinical outcomes in patients with metastatic CRC. Overall survival was calculated from the date of treatment initiation to the date of death from any cause. Progression-free survival (PFS) was defined as the time interval from the date of treatment initiation to the date of disease progression or death from any cause. Categorical variables were compared using Pearson's $\chi^{2}$ test or Fisher's exact test. The Mantel-Haenszel linear-by-linear association $\chi^{2}$ test was used to analyse the linear trend of ordinal and categorical variables according to the CIMP status. For estimation of OS and PFS, the Kaplan-Meier method was used, and comparisons were made using log-rank tests. To adjust for baseline characteristics, we performed multivariable analysis with a Cox proportional hazard model using a backward stepwise elimination approach. Age (continuous variable), sex, differentiation (well-differentiated to moderately differentiated $v s$ poorly differentiated), tumour location 
(proximal $v s$ distal), number of metastatic organs $(1-2 v s \geqslant 3)$, Eastern Cooperative Oncology Group (ECOG) performance status $(0 v s \geqslant 1)$, serum carcinoembryonic antigen (CEA) level ( $\leqslant 5$ vs $>5 \mathrm{ng} \mathrm{ml}^{-1}$ ), BRAF mutation status, and MSI status (MSI-high vs MSI-low/MSS) were included as covariates on the basis of previous studies on metastatic CRC (Bae et al, 2013; Park et al, 2013). Twosided $P$-values of $<0.05$ were considered statistically significant. All statistical analyses were performed using IBM SPSS Statistics for Windows, version 20.0 (IBM Corp., Armonk, NY, USA). This study was analysed and reported according to the REporting recommendations for tumour MARKer prognostic studies (REMARK) (McShane et al, 2005).

\section{RESULTS}

Patient characteristics. A total of 153 patients were included (Figure 1). Tumour specimens were collected from August 2008 to April 2013. The patient characteristics are described in Table 1. The primary tumour locations included the caecum in seven patients, ascending colon in 26, transverse colon in nine, descending colon in 10, sigmoid colon in 62, and rectum in 39. Collectively, 42 patients (27.5\%) exhibited proximal (caecum to transverse colon) lesions and $112(72.5 \%)$ exhibited distal lesions. In total, $138(90.2 \%)$ patients were diagnosed with initial metastatic disease and 15 (9.8\%) with recurrent disease. According to the inclusion criteria, all patients had undergone resection of the primary tumour. Primary tumour resection and tissue sampling were performed before the initiation of palliative chemotherapy in 132 patients with initial metastatic disease and after the initiation of chemotherapy in the remaining 6 patients. In addition, 15 patients with recurrent disease had undergone curative surgery for the primary tumour, 12 of whom also received adjuvant chemotherapy.

The first-line chemotherapy regimens included fluoropyrimidine and oxaliplatin in 128 patients (FOLFOX in 114, XELOX in 11 , and SOX in three), FOLFIRI in 18, and capecitabine in seven. Bevacizumab or cetuximab was combined for five and two patients, respectively. Second-line chemotherapy was administered to 119
(77.8\%) patients. As second-line agents, fluoropyrimidine and irinotecan were used for 86 patients (FOLFIRI in 84 and XELIRI in 2 ), fluoropyrimidine and oxaliplatin for 28 (FOLFOX in 25 and XELOX in 3), and capecitabine for 5. Bevacizumab or cetuximab was added for five and four patients, respectively.

The median follow-up duration after the diagnosis of metastatic CRC was 41.8 months [95\% confidence interval (CI), 36.8-46.8]. At the time of the last follow-up (November 2014), 103 patients $(67.3 \%)$ had succumbed, and the median OS was 27.0 months (95\% CI, 22.4-31.6).

CIMP status and clinicopathological characteristics. Methylation was observed at $\geqslant 1$ loci in $76(49.7 \%)$ patients. In all, 7 (4.6\%) patients exhibited $\geqslant 5$ methylated loci (CIMP-high), 69 (45.1\%) exhibited 1-4 methylated loci (CIMP-low), and 77 (50.3\%) exhibited no methylated loci (CIMP-negative). CDKN2A was the most frequently methylated locus (60 patients; $39.2 \%$ ), followed by CRABP1 (34; 22.2\%), NEUROG1 (20;13.1\%), IGF2 (12; 7.8\%), SOCS1 (10;6.5\%), CACNA1G (9; 5.9\%), RUNX3 (7; 4.6\%), and MLH1 (1;0.7\%). Methylation was more frequently observed in tumours with a proximal location, poor differentiation, BRAF mutations, and an MSI-high status (Table 1). There was no significant difference in the methylation status according to the number of metastatic organs or the timing of primary tumour resection.

CIMP status and clinical outcomes. Overall survival after the initiation of palliative chemotherapy was significantly different among the three CIMP groups $(P<0.001$; Figure 2$)$. The median OS was 35.7 months (95\% CI, 30.5-40.9) for the CIMP-negative group, 22.2 months (95\% CI, 17.2-27.2) for the CIMP-low group, and 9.77 months (95\% CI, 2.07-17.5) for the CIMP-high group. Multivariable analysis revealed that the adjusted hazard ratios (aHRs) for OS were 2.29 (95\% CI, 1.50-3.49) and 11.3 (95\% CI, 4.72-26.8) for the CIMP-low and CIMP-high groups, respectively (both $P<0.001$; Table 2).

To increase homogeneity, patients treated with fluoropyrimidine and oxaliplatin first-line chemotherapy $(N=128)$ were analysed. In line with the overall population, OS was significantly better for the CIMP-negative group $(P<0.001$; Figure $3 \mathrm{~A})$, with

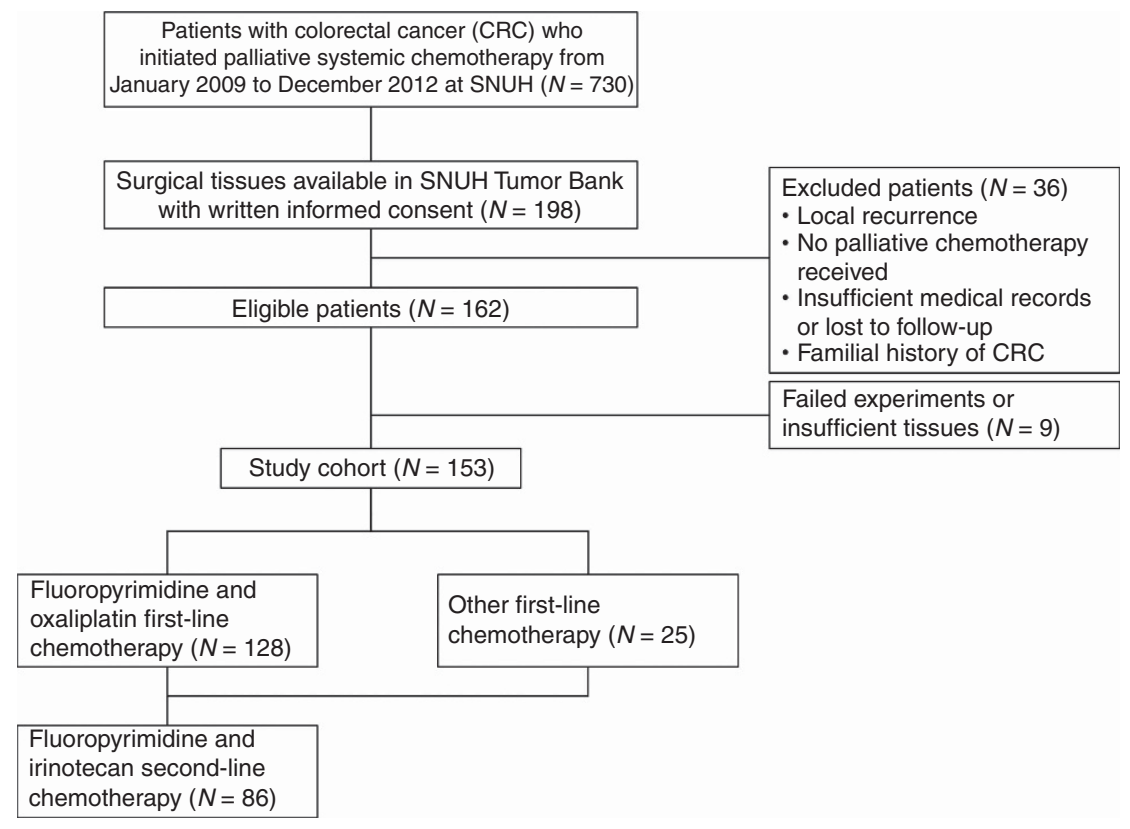

Figure 1. REporting recommendations for tumour MARKer prognostic studies (REMARK) diagram describes flow of the study cohort, including number of patients included in each stage of the study (McShane et al, 2005). SNUH, Seoul National University Hospital. 
Table 1. Patient characteristics and the CIMP status

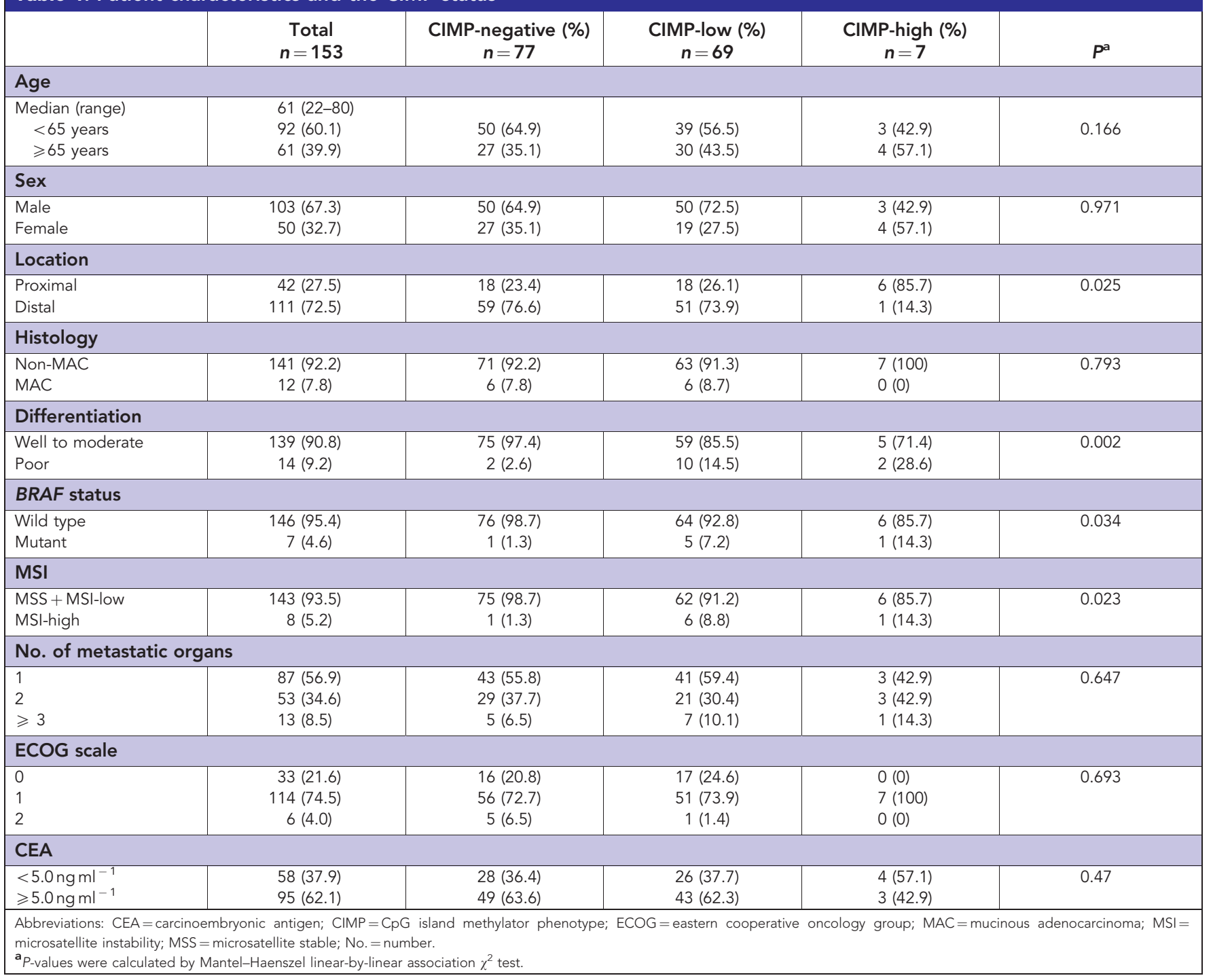

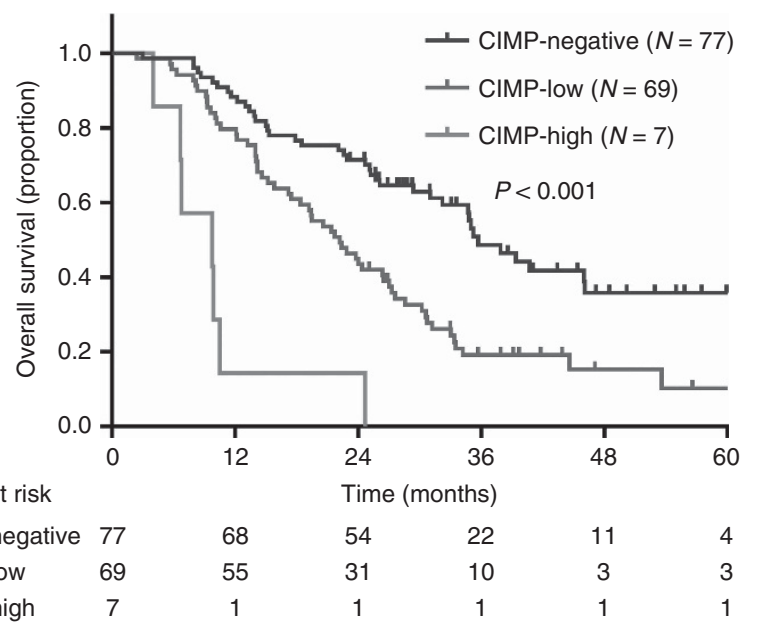

Figure 2. Kaplan-Meier curves for overall survival (OS) according to the $\mathrm{CpG}$ island methylator phenotype (CIMP) status in patients with metastatic colorectal cancer treated with chemotherapy. OS was significantly different among the three CIMP groups, with a median of 35.7 months for the negative group, 22.2 months for the low group, and 9.77 months for the high group $(P<0.001)$. median values of 37.9 (95\% CI, 31.6-44.2), 23.8 (95\% CI, 17.929.7), and 6.77 (95\% CI, 3.09-10.5) months for the CIMP-negative, CIMP-low, and CIMP-high groups, respectively. Similar results were observed for PFS, with higher survival values derived for the CIMP-negative group $(P=0.002$; Figure $3 \mathrm{~B})$. The median PFS was 9.97 (95\% CI, 8.10-11.8), 7.87 (95\% CI, 5.95-9.79), and 1.83 (95\% CI, 1.55-2.11) months for the CIMP-negative, CIMP-low, and CIMP-high groups, respectively. RR showed a marginal difference according to the CIMP status $(P=0.107)$ : $53.4 \%, 45.1 \%$, and $16.7 \%$, respectively. The significant differences in OS and PFS among the three CIMP groups of patients treated with fluoropyrimidine and oxaliplatin first-line chemotherapy were maintained after adjusting for potential confounding variables in multivariable analysis (Table 3). Adjusted hazard ratios for OS were 2.34 (1.453.76) and $11.9(4.62-30.8)$ for the CIMP-low and CIMP-high groups, respectively (both $P<0.001$ ), while those for PFS were 1.82 $(1.17-2.84 ; P=0.008)$ and $3.53(1.47-8.48 ; P=0.005)$, respectively.

For patients treated with fluoropyrimidine and irinotecan second-line chemotherapy $(N=86)$, OS following second-line therapy was significantly different among the three CIMP groups $(P<0.001$; Figure 3C), with median values of 20.4 (95\% CI, 6.0634.7), 13.4 (95\% CI, 12.4-14.4), and 2.90 (95\% CI, 0.173-5.63) months for the CIMP-negative, CIMP-low, and CIMP-high groups, respectively. However, PFS showed no differences 
$(P=0.226$; Figure 3D), with median values of 4.83 (95\% CI, 3.666.00), 4.40 (95\% CI, 2.13-6.67), and 1.63 (95\% CI, 0.278-2.98) months, respectively. RR also showed no differences $(P=0.482)$,

Table 2. Multivariable analysis of overall survival in the overall population

\begin{tabular}{|l|c|c|}
\cline { 2 - 3 } & \multicolumn{2}{c|}{ Overall survival } \\
\hline $\begin{array}{l}\text { Covariates in the final } \\
\text { model }\end{array}$ & Adjusted HR (95\% Cl) & $P$ \\
\hline Age & $1.02(1.01-1.04)$ & 0.013 \\
\hline Female $(N=49)$ & $1.70(1.10-2.62)$ & 0.017 \\
\hline ECOG $\geqslant 1(N=119)$ & $1.88(1.06-3.36)$ & 0.032 \\
\hline Metastatic organ $\geqslant 3(N=11)$ & $5.27(2.50-11.1)$ & $<0.001$ \\
\hline CIMP-high $(N=7)$ & $11.3(4.72-26.8)$ & $<0.001$ \\
\hline CIMP-low $(N=68)$ & $2.29(1.50-3.49)$ & $<0.001$ \\
\hline $\begin{array}{l}\text { Abbreviations: } \mathrm{Cl} \text {, confidence interval; } \mathrm{CIMP}=\mathrm{CpG} \text { island methylator phenotype; } \mathrm{ECOG}= \\
\text { Eastern Cooperative Oncology Group; } \mathrm{HR}=\text { hazard ratio. }\end{array}$ \\
\hline
\end{tabular}

A

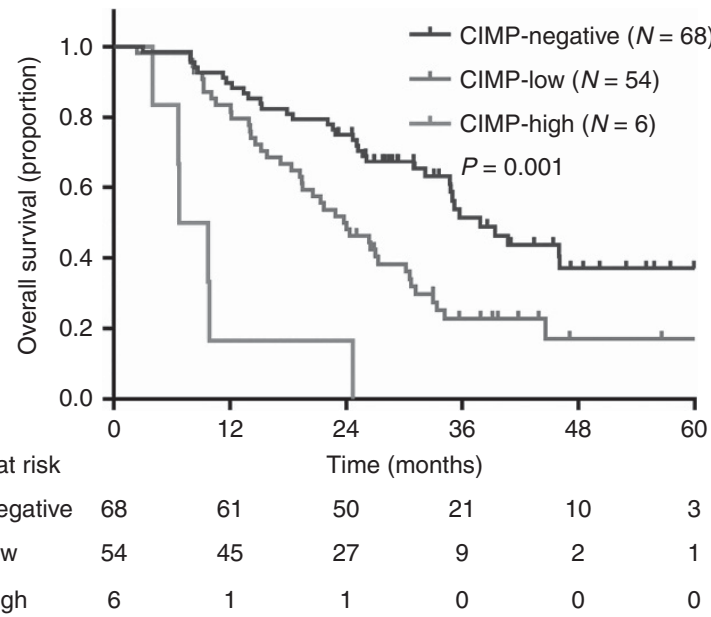

C

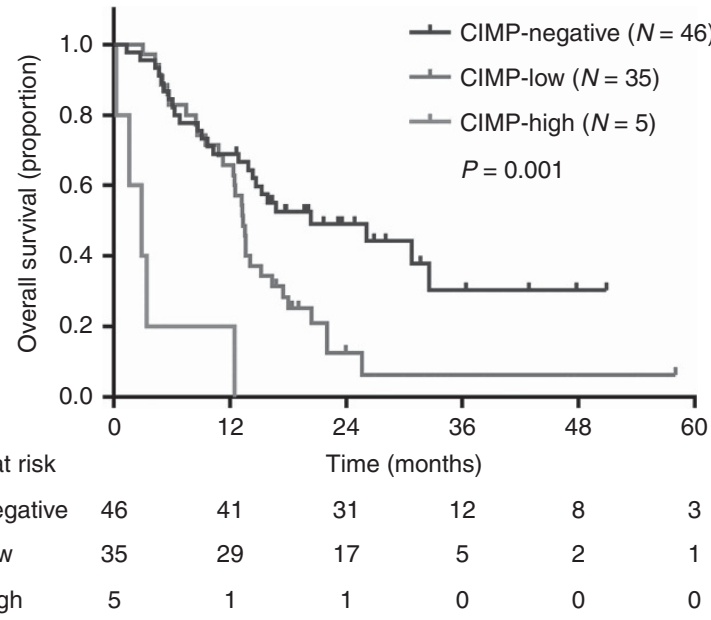

with values of $11.6 \%, 20.6 \%$, and $0.0 \%$, respectively. In multivariable analysis, OS showed a significant difference according to the CIMP status (Table 3), with aHRs of 2.25 (95\% CI, 1.28-3.94; $P=0.005)$ and 29.1 (95\% CI, 9.01-94.0; $P<0.001)$ for the CIMPlow and CIMP-high groups, respectively.

In addition to the CIMP status, we analysed clinical outcomes with regard to the methylation status at each locus. MLH1 was excluded, because only one $(0.7 \%)$ patient showed positive methylation. Methylation at all individual loci except SOCS1 and RUNX3 (CACNA1G, IGF2, NEUROG1, CDKN2A, and CRABP1) was associated with shorter OS in multivariable analysis. Notably, methylation at IGF2 and NEUROG1 was associated with significantly shorter OS and PFS in patients treated with fluoropyrimidine plus oxaliplatin first-line chemotherapy and those treated with fluoropyrimidine plus irinotecan second-line chemotherapy (Supplementary Material 2).

We also analysed clinical outcomes with the number of methylated CIMP loci as a continuous variable in multivariable analysis. The higher number of methylated loci was significantly associated with shortened OS and PFS in the overall population, as well as among

B

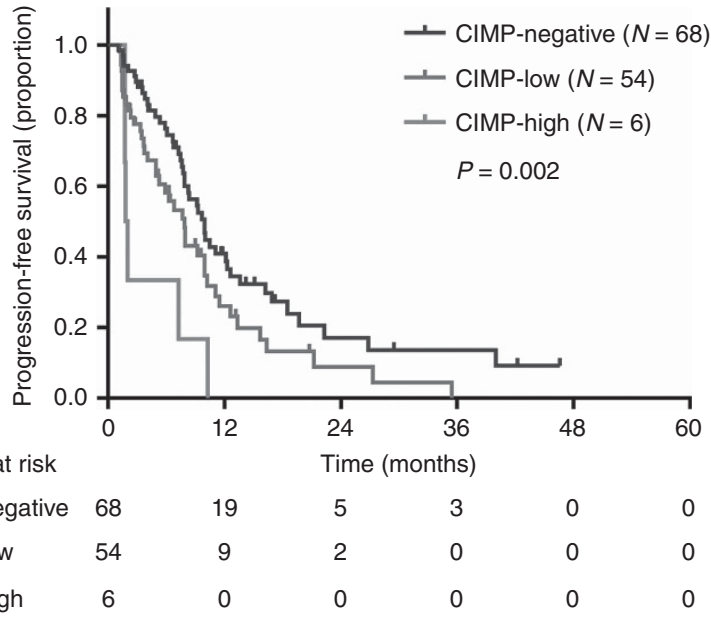

D

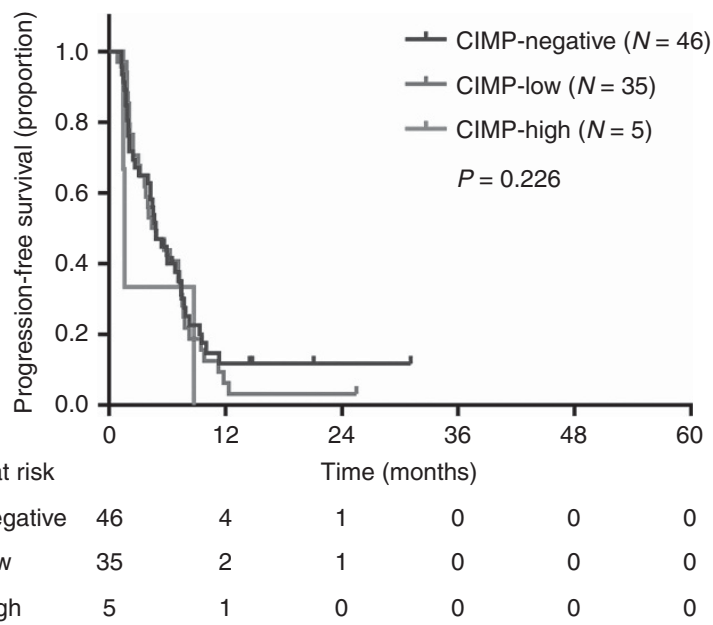

Figure 3. Kaplan-Meier curves for overall survival and progression-free survival according to the CpG island methylator phenotype (CIMP) status in patients treated with fluoropyrimidine plus oxaliplatin first-line chemotherapy (A and $\mathbf{B})$ and fluoropyrimidine plus irinotecan secondline chemotherapy (C and D) for metastatic colorectal cancer. For patients treated with fluoropyrimidine and oxaliplatin first-line chemotherapy, OS and PFS were significantly different among the three CIMP groups (median OS 37.9 vs 23.8 vs 6.77 months for the negative, low, and high groups, $P<0.001$; median PFS 9.97 vs 7.87 vs 1.83 months for the negative, low, and high groups, respectively, $P=0.002$ ). For patients treated with fluoropyrimidine and irinotecan second-line chemotherapy, only OS was different among the three CIMP groups (median OS 20.4 vs 13.4 vs 2.90 months for the negative, low, and high groups, $P<0.001$; median PFS 4.83 vs 4.40 vs 1.63 months for the negative, low, and high groups, respectively, $P=0.226$ ). 
Table 3. Multivariable analysis of overall and progression-free survival in patients treated with fluoropyrimidine plus oxaliplatin first-line chemotherapy and fluoropyrimidine plus irinotecan second-line chemotherapy

\begin{tabular}{|c|c|c|c|c|c|}
\hline \multicolumn{4}{|c|}{ Overall survival } & \multicolumn{2}{|c|}{ Progression-free survival } \\
\hline Covariates in the final model & Adjusted HR (95\% Cl) & $P$ & $\begin{array}{l}\text { Covariates in the final } \\
\text { model }\end{array}$ & Adjusted HR (95\% Cl) & $P$ \\
\hline \multicolumn{6}{|c|}{ Fluoropyrimidine and oxaliplatin first-line chemotherapy $(N=128)$} \\
\hline $\begin{array}{l}\text { Age } \\
\text { Female }(N=38) \\
\text { ECOG } \geqslant 1(N=96) \\
\text { Metastatic organ } \geqslant 3(N=9) \\
\text { CIMP-high }(N=6) \\
\text { CIMP-low }(N=54)\end{array}$ & $\begin{array}{l}1.02(1.00-1.05) \\
1.60(0.959-2.66) \\
1.70(0.929-3.09) \\
5.41(2.36-12.4) \\
11.9(4.62-30.8) \\
2.34(1.45-3.76)\end{array}$ & $\begin{array}{r}0.034 \\
0.072 \\
0.085 \\
<0.001 \\
<0.001 \\
<0.001\end{array}$ & $\begin{array}{c}\text { ECOG } \geqslant 1(N=96) \\
\text { Metastatic organ } \geqslant 3(N=9) \\
\text { CIMP-high }(N=6) \\
\text { CIMP-low }(N=54)\end{array}$ & $\begin{array}{l}1.95(1.15-3.33) \\
2.46(1.10-5.48) \\
3.53(1.47-8.48) \\
1.82(1.17-2.84)\end{array}$ & $\begin{array}{l}0.013 \\
0.028 \\
0.005 \\
0.008\end{array}$ \\
\hline \multicolumn{6}{|c|}{ Fluoropyrimidine and irinotecan second-line chemotherapy $(\mathbf{N}=86)$} \\
\hline $\begin{array}{l}\text { Female }(N=30) \\
\text { Metastatic organ } \geqslant 3(N=6) \\
\text { CEA } \geqslant 5.0 \mathrm{ng} \mathrm{ml}^{-1}(N=54) \\
\text { CIMP-high }(N=5) \\
\text { CIMP-low }(N=35)\end{array}$ & $\begin{array}{l}1.85(1.03-3.34) \\
3.70(1.39-9.83) \\
2.27(1.22-4.24) \\
29.1(9.01-94.0) \\
2.25(1.28-3.94)\end{array}$ & $\begin{aligned} & 0.04 \\
& 0.009 \\
& 0.01 \\
< & 0.001 \\
& 0.005\end{aligned}$ & $\begin{array}{c}\text { Female }(N=30) \\
\text { ECOG } \geqslant 1(N=66) \\
\text { CEA } \geqslant 5.0 \mathrm{ng} \mathrm{ml}^{-1}(N=54) \\
\text { MSS/MSI-low }(N=81) \\
\text { CIMP-high }(N=5) \\
\text { CIMP-low }(N=35)\end{array}$ & $\begin{array}{l}2.06(1.21-3.48) \\
1.80(0.991-3.26) \\
1.89(1.11-3.24) \\
3.81(0.86-16.9) \\
3.50(1.16-10.6) \\
1.39(0.841-2.28)\end{array}$ & $\begin{array}{l}0.007 \\
0.054 \\
0.02 \\
0.078 \\
0.027 \\
0.2\end{array}$ \\
\hline
\end{tabular}

patients treated with oxaliplatin based first- and irinotecan-based second-line chemotherapy (Supplementary Material 3).

\section{DISCUSSION}

In the present study, we aimed to assess the clinical implications of the CIMP status in patients with metastatic CRC treated with systemic chemotherapy. For this purpose, quantitative analyses using MethyLight technology were performed to evaluate DNA methylation in eight markers (CACNA1G, IGF2, NEUROG1, RUNX3, SOCS1, CDKN2A, CRABP1, and MLH1) originally proposed by Ogino et al (2006a, 2007a). The presence of $\geqslant 5$ methylated promoters was considered to represent a CIMP-high status, because the clinicopathological characteristics of CIMP-high tumours were shown to have the best correlation with a cutoff value of 5 in our previous studies (Kim et al, 2009; Bae et al, 2011, 2013).

In the present study, the CIMP status was associated with adverse clinical outcomes in metastatic CRC, and this prognostic impact was maintained with first- and second-line chemotherapy regimens. To the best of our knowledge, this is the largest study evaluating the relationship between the CIMP status and clinical outcomes in metastatic CRC treated with chemotherapy. In a smaller study including 30 previously untreated metastatic tumours treated with first-line chemotherapy with irinotecan, leucovorin, 5-fluorouracil, and gefitinib, a CIMP-high status was associated with a decreased median survival duration (0.9 vs 1.9 years) (Ogino et al, 2007b). When analysed by individual markers, methylation of CACNA1G, IGF2, NEUROG1, RUNX3, MLH1, MINT31, and WRN was associated with poor survival, while that of CDKN2A, CRABP1, SOCS1, MINT1, IGFBP3, and MGMT was not. Although this study included only a small number of patients with metastatic CRC treated with unconventional chemotherapy regimens, the findings are consistent with those of the present study. The finding of a negative prognostic effect of the CIMP status in the present study is also consistent with the findings of the recent meta-analysis mentioned earlier and suggests that the adverse influence of the CIMP status on clinical outcomes also applies to metastatic CRC (Juo et al, 2014). Because our study includes the largest and most homogeneous population of patients with metastatic CRC treated with standard chemotherapy regimens, we believe our findings provide the most clinically significant evidence on the prognostic implications of CIMP in metastatic CRC.

In the present study, we further investigated the treatment outcomes in terms of OS, PFS, and objective response in patient groups treated with oxaliplatin-based first-line and irinotecanbased second-line treatments to determine the influence of the CIMP status in different treatment settings. For patients treated with fluoropyrimidine and oxaliplatin first-line chemotherapy, the CIMP status was associated with decreased OS and PFS and a trend towards non-response. For patients treated with fluoropyrimidine and irinotecan second-line treatment, the CIMP status was correlated with poor OS and a trend for poor PFS. Although our study analysed a single treatment arm, making it difficult to determine whether the negative impact of the CIMP status on treatment outcomes is prognostic or predictive in nature, an apparently stronger association in patients treated with oxaliplatinbased chemotherapy regimens than in those treated with irinotecan-based chemotherapy regimens suggests that the CIMP status may interact with the chemotherapy regimen. In a recent exploratory analysis of patients with stage III CRC treated with 5fluorouracil and leucovorin alone (FL) or irinotecan (IFL), CIMPpositive patients treated with IFL showed a trend towards increased OS compared with those treated with FL (Shiovitz et al, 2014). The potential interaction between the CIMP status and chemotherapy should be assessed in future studies with multiple treatment arms.

CIMP is believed to promote carcinogenesis through methylationmediated transcriptional silencing in tumour suppressor genes (Coppede, 2014). However, the role of CIMP in the pathogenesis of $\mathrm{CRC}$ and the mechanism underlying its prognostic and/or predictive influence remain largely unknown. Whether abnormal global DNA methylation or local methylation in some CIMP loci is responsible for different outcomes remains unclear. In this context, our finding that individual CIMP loci as well as the CIMP status are associated with treatment outcomes can be interesting. The stronger association of methylated IGF2 and NEUROG1 loci with treatment outcomes compared with that of other loci in the present study deserves further investigation focused on a mechanistic understanding.

In conclusion, the results of our study suggest that the CIMP status is associated with unfavourable clinical outcomes in patients with metastatic CRC treated with chemotherapy, and that the negative prognostic impact is maintained throughout first- and second-line chemotherapy regimens. 


\section{ACKNOWLEDGEMENTS}

This research was supported by grants from the Basic Science Research Program through the National Research Foundation of Korea (NRF) funded by the Ministry of Education (2013R1A1A2059080), the Korea Health Technology R\&D Project (HI13C1804 and HI14C1277) through the Korea Health Industry Development Institute (KHIDI) funded by the Ministry of Health and Welfare, and the Converging Research Center Program funded by the Ministry of Science, ICT and Future Planning, Republic of Korea (2014M3C1A8048802).

\section{CONFLICT OF INTEREST}

The authors declare no conflict of interest.

\section{REFERENCES}

Bae JM, Kim JH, Cho NY, Kim TY, Kang GH (2013) Prognostic implication of the $\mathrm{CpG}$ island methylator phenotype in colorectal cancers depends on tumour location. Br J Cancer 109(4): 1004-1012.

Bae JM, Kim MJ, Kim JH, Koh JM, Cho NY, Kim TY, Kang GH (2011) Differential clinicopathological features in microsatellite instabilitypositive colorectal cancers depending on CIMP status. Virchows Arch 459(1): 55-63.

Coppede F (2014) Epigenetic biomarkers of colorectal cancer: focus on DNA methylation. Cancer Lett 342(2): 238-247.

Han SW, Lee HJ, Bae JM, Cho NY, Lee KH, Kim TY, Oh DY, Im SA, Bang YJ, Jeong SY, Park KJ, Park JG, Kang GH, Kim TY (2013) Methylation and microsatellite status and recurrence following adjuvant FOLFOX in colorectal cancer. Int J Cancer 132(9): 2209-2216.

Hughes LA, Melotte V, de Schrijver J, de Maat M, Smit VT, Bovee JV, French PJ, van den Brandt PA, Schouten LJ, de Meyer T, van Criekinge W, Ahuja N, Herman JG, Weijenberg MP, van Engeland M (2013) The CpG island methylator phenotype: what's in a name? Cancer Res 73(19): 5858-5868.

Iacopetta B, Kawakami K, Watanabe T (2008) Predicting clinical outcome of 5-fluorouracil-based chemotherapy for colon cancer patients: is the $\mathrm{CpG}$ island methylator phenotype the 5-fluorouracil-responsive subgroup? Int Clin Oncol 13(6): 498-503.

Jover R, Nguyen TP, Perez-Carbonell L, Zapater P, Paya A, Alenda C, Rojas E, Cubiella J, Balaguer F, Morillas JD, Clofent J, Bujanda L, Rene JM, Bessa X, Xicola RM, Nicolas-Perez D, Castells A, Andreu M, Llor X, Boland CR, Goel A (2011) 5-Fluorouracil adjuvant chemotherapy does not increase survival in patients with $\mathrm{CpG}$ island methylator phenotype colorectal cancer. Gastroenterology 140(4): 1174-1181.

Juo YY, Johnston FM, Zhang DY, Juo HH, Wang H, Pappou EP, Yu T, Easwaran H, Baylin S, van Engeland M, Ahuja N (2014) Prognostic value of $\mathrm{CpG}$ island methylator phenotype among colorectal cancer patients: a systematic review and meta-analysis. Ann Oncol 25(12): 2314-2327.

Kang GH, Lee S, Cho NY, Gandamihardja T, Long TI, Weisenberger DJ, Campan M, Laird PW (2008) DNA methylation profiles of gastric carcinoma characterized by quantitative DNA methylation analysis. Lab Investig 88(2): 161-170.

Kim JE, Hong YS, Ryu MH, Lee JL, Chang HM, Lim SB, Kim JH, Jang SJ, Kim MJ, Yu CS, Kang YK, Kim JC, Kim TW (2011) Association between deficient mismatch repair system and efficacy to irinotecancontaining chemotherapy in metastatic colon cancer. Cancer Sci 102(9): 1706-1711.

Kim JH, Kang GH (2014) Molecular and prognostic heterogeneity of microsatellite-unstable colorectal cancer. World J Gastroenterol 20(15): 4230-4243.

Kim JH, Shin SH, Kwon HJ, Cho NY, Kang GH (2009) Prognostic implications of $\mathrm{CpG}$ island hypermethylator phenotype in colorectal cancers. Virchows Arch 455(6): 485-494.

Lee DW, Kim KJ, Han SW, Lee HJ, Rhee YY, Bae JM, Cho NY, Lee KH, Kim TY, Oh DY, Im SA, Bang YJ, Jeong SY, Park KJ, Park JG, Kang GH,
Kim TY (2015) KRAS mutation is associated with worse prognosis in stage III or high-risk stage II colon cancer patients treated with adjuvant FOLFOX. Ann Surg Oncol 22(1): 187-194.

Martinez-Garcia R, Lopez-Casas PP, Rico D, Valencia A, Hidalgo M (2014) Colorectal cancer classification based on gene expression is not associated with FOLFIRI response. Nat Med 20(11): 1230-1231.

McShane LM, Altman DG, Sauerbrei W, Taube SE, Gion M, Clark GM (2005) REporting recommendations for tumour MARKer prognostic studies (REMARK). Br J Cancer 93(4): 387-391.

Min BH, Bae JM, Lee EJ, Yu HS, Kim YH, Chang DK, Kim HC, Park CK, Lee SH, Kim KM, Kang GH (2011) The CpG island methylator phenotype may confer a survival benefit in patients with stage II or III colorectal carcinomas receiving fluoropyrimidine-based adjuvant chemotherapy. BMC Cancer 11: 344.

Ogino S, Cantor M, Kawasaki T, Brahmandam M, Kirkner GJ, Weisenberger DJ, Campan M, Laird PW, Loda M, Fuchs CS (2006a) CpG island methylator phenotype (CIMP) of colorectal cancer is best characterised by quantitative DNA methylation analysis and prospective cohort studies. Gut 55(7): 1000-1006.

Ogino S, Kawasaki T, Brahmandam M, Cantor M, Kirkner GJ, Spiegelman D, Makrigiorgos GM, Weisenberger DJ, Laird PW, Loda M, Fuchs CS (2006b) Precision and performance characteristics of bisulfite conversion and real-time PCR (MethyLight) for quantitative DNA methylation analysis. J Mol Diagn 8(2): 209-217.

Ogino S, Kawasaki T, Kirkner GJ, Kraft P, Loda M, Fuchs CS (2007a) Evaluation of markers for $\mathrm{CpG}$ island methylator phenotype (CIMP) in colorectal cancer by a large population-based sample. J Mol Diagn 9(3): 305-314.

Ogino S, Meyerhardt JA, Kawasaki T, Clark JW, Ryan DP, Kulke MH, Enzinger PC, Wolpin BM, Loda M, Fuchs CS (2007b) CpG island methylation, response to combination chemotherapy, and patient survival in advanced microsatellite stable colorectal carcinoma. Virchows Arch 450(5): 529-537.

Ogino S, Nosho K, Kirkner GJ, Kawasaki T, Meyerhardt JA, Loda M, Giovannucci EL, Fuchs CS (2009) CpG island methylator phenotype, microsatellite instability, BRAF mutation and clinical outcome in colon cancer. Gut 58(1): 90-96.

Park JH, Kim TY, Lee KH, Han SW, Oh DY, Im SA, Kang GH, Chie EK, Ha SW, Jeong SY, Park KJ, Park JG, Kim TY (2013) The beneficial effect of palliative resection in metastatic colorectal cancer. Br J Cancer 108(7): $1425-1431$

Sadanandam A, Gray J, Hanahan D (2014) Reply to Colorectal cancer classification based on gene expression is not associated with FOLFIRI response. Nat Med 20(11): 1231-1232.

Sadanandam A, Lyssiotis CA, Homicsko K, Collisson EA, Gibb WJ, Wullschleger S, Ostos LC, Lannon WA, Grotzinger C, Del Rio M, Lhermitte B, Olshen AB, Wiedenmann B, Cantley LC, Gray JW, Hanahan D (2013) A colorectal cancer classification system that associates cellular phenotype and responses to therapy. Nat Med 19(5): 619-625.

Shen L, Catalano PJ, Benson 3rd AB, O’Dwyer P, Hamilton SR, Issa JP (2007) Association between DNA methylation and shortened survival in patients with advanced colorectal cancer treated with 5 -fluorouracil based chemotherapy. Clin Cancer Res 13(20): 6093-6098.

Shiovitz S, Bertagnolli MM, Renfro LA, Nam E, Foster NR, Dzieciatkowski S, Luo Y, Lao VV, Monnat Jr RJ, Emond MJ, Maizels N, Niedzwiecki D, Goldberg RM, Saltz LB, Venook A, Warren RS, Grady WM. Alliance for Clinical Trials in Oncology (2014) CpG island methylator phenotype is associated with response to adjuvant irinotecan-based therapy for stage III colon cancer. Gastroenterology 147(3): 637-645.

Siegel R, Ma J, Zou Z, Jemal A (2014) Cancer statistics, 2014. CA Cancer J Clin 64(1): 9-29.

Sistigu A, Yamazaki T, Vacchelli E, Chaba K, Enot DP, Adam J, Vitale I, Goubar A, Baracco EE, Remedios C, Fend L, Hannani D, Aymeric L, Ma Y, Niso-Santano M, Kepp O, Schultze JL, Tuting T, Belardelli F, Bracci L, La Sorsa V, Ziccheddu G, Sestili P, Urbani F, Delorenzi M, Lacroix-Triki M, Quidville V, Conforti R, Spano JP, Pusztai L, Poirier-Colame V, Delaloge S, Penault-Llorca F, Ladoire S, Arnould L, Cyrta J, Dessoliers MC, Eggermont A, Bianchi ME, Pittet M, Engblom C, Pfirschke C, Preville X, Uze G, Schreiber RD, Chow MT, Smyth MJ, Proietti E, Andre F, Kroemer G, Zitvogel L (2014) Cancer cell-autonomous contribution of type I interferon signaling to the efficacy of chemotherapy. Nat Med 20(11): 1301-1309. 
Van Rijnsoever M, Elsaleh H, Joseph D, McCaul K, Iacopetta B (2003) CpG island methylator phenotype is an independent predictor of survival benefit from 5 -fluorouracil in stage III colorectal cancer. Clin Cancer Res 9(8): 2898-2903.

Weisenberger DJ, Siegmund KD, Campan M, Young J, Long TI, Faasse MA, Kang GH, Widschwendter M, Weener D, Buchanan D, Koh H, Simms L, Barker M, Leggett B, Levine J, Kim M, French AJ, Thibodeau SN, Jass J, Haile R, Laird PW (2006) CpG island methylator phenotype underlies sporadic microsatellite instability and is tightly associated with BRAF mutation in colorectal cancer. Nat Genet 38(7): 787-793.

This work is published under the standard license to publish agreement. After 12 months the work will become freely available and the license terms will switch to a Creative Commons AttributionNonCommercial-Share Alike 4.0 Unported License.

Supplementary Information accompanies this paper on British Journal of Cancer website (http://www.nature.com/bjc) 